九州大学学術情報リポジトリ

Kyushu University Institutional Repository

\title{
Reduction of crosstalk noise by optimizing 3-D configuration of the routing grid
}

Sakai, Atsushi

Materials and Devices Development Center SANYO Electric Co., Ltd.

Yamada, Takashi

Materials and Devices Development Center SANYO Electric Co., Ltd.

Matsushita, Yoshifumi

Materials and Devices Development Center SANYO Electric Co., Ltd.

Yasuura, Hiroto

Department of Computer Science and Communication Engineering, Kyushu University

http://hdl. handle. net/2324/6027

出版情報: Proc. of the Asia and South Pacific Design Automation Conference (ASP-DAC 2003), pp. 49-52, 2003-01. IEEE

バージョン:

権利関係 : 


\section{Reduction of crosstalk noise by optimizing 3-D configuration of the routing grid}

\author{
Atsushi Sakai, Takashi Yamada, \\ Yoshifumi Matsushita
Materials and Devices Development Center
SANYO Electric Co., Ltd.
180 Ohmori, Anpachi-cho, Anpachi-gun, Gifu 503-0195, Japan \\ Tel: +81-584-64-5218 Fax: +81-584-64-5214 \\ e-mail: \{sakai, yamada, matusita\}@ul.rd.sanyo.co.jp
}

\author{
Department of Computer Science and \\ Communication Engineering, Kyushu University \\ 6-1 Kasuga-koen, Kasuga, \\ Fukuoka 816-8580, Japan \\ Tel: +81-92-583-7620 Fax: +81-92-583-1338 \\ e-mail: yasuura@c.csce.kyushu-u.ac.jp
}

\begin{abstract}
In this paper, we propose novel physical design techniques for a sub-quarter micron system-on-a-chip (SoC). By appropriately optimizing the routing grid space or the cell utilization ratio, the coupling effects are almost eliminated. By employing our proposed techniques on a $0.13 \mu \mathrm{m}$ six-layer physical design, the longest path delay is significantly decreased by $15 \%$ maximum without the need for process improvement. This significant delay reduction, which corresponds to a half generation of process progress, greatly accelerates the performance of SoCs.
\end{abstract}

\section{Introduction}

In the case of SoCs fabricated in sub-quarter micron or later nanometer-scale technologies, the interconnect delay is an important performance limiting factor [1]. The proportion of gate and line-to-substrate capacitance to the overall capacitance decreases while that of line-to-line capacitance increases. Therefore, on-chip crosstalk is now becoming manifest $[2,3,4,5]$. This causes logic errors [6] and delay variations because of signal distortion.

Another unwanted phenomenon of crosstalk is the large variation in effective line-to-line capacitance. If opposite-direction switching activity occurs in adjacent lines, the effective line-to-line capacitance will be doubled. By contrast, if same-direction switching activity occurs in adjacent lines, the line-to-line capacitance is considered to be nonexistent [7]. Because it is difficult to identify the state of the switching activity occurring between adjacent lines, timing constraints must be satisfied in both opposite- and same-direction switching. Therefore, timing closure becomes even more difficult if significant crosstalk noise occurs.

To reduce these unwanted effects, circuitry techniques have been proposed. In the cases reported in $[8,9]$, crosstalk noise was reduced by inserting repeaters. In the case reported in [6], the variations in effective line-to-line capacitance were mitigated by inserting repeaters (inverters) between adjacent lines at staggered points. The circuitry changes would have a direct effect on timing and might result in an increased chip area. Insertion of repeaters at staggered points is effective mainly in making a handcrafted or semi-custom design. However, it is difficult to locate the most suitable insertion points if using an automatic place and route methodology.

Routing techniques were also proposed. One technique is to change the line width and space without changing the given line pitch $[10,11]$. By using this technique, line capacitance or line resistance change considerably when the line widths are greatly widened and as a result the timing is unfavorably affected. Another technique is to increase the distance to adjacent lines for chosen critical nets [12]. By using this method, the degree of congestion in un-chosen nets increases and as a result the overall crosstalk noise cannot be decreased. Another different technique is to establish a power line and other lines that have no electric-potential variations on either side of a noise-sensitive line for shielding [10,13,14]. By using this method, many wiring resources are required for shielding and the design complexity is also increased.

To solve these drawbacks, we propose new physical design techniques by simply optimizing the routing grid space or the cell utilization ratio for the automatic place and route methodology that is common in today's large-scale SoC design. The correlation between crosstalk noise and the layout-related settings are investigated for developing our techniques.

\section{Techniques to Reduce Crosstalk}

Line-to-line capacitive coupling between signal wires, which is the main cause of crosstalk noise, can be separated into two components, which are a horizontal line-to-line capacitance $\left(\mathrm{C}_{\mathrm{H}}\right)$ and a vertical line-to-line capacitance $\left(\mathrm{C}_{\mathrm{V}}\right)$. In the case reported in [15], the ratio of $C_{H}$ to $C_{V}$ is expressed as follows:

$$
\mathrm{C}_{\mathrm{H}} / \mathrm{C}_{\mathrm{V}}=(\mathrm{AR})^{\mathrm{n}}: \quad \mathrm{n} \in[1,2]
$$

where $\mathrm{AR}$ is the aspect ratio of the lines (the ratio of the length of line cross sections in the vertical direction to that in the horizontal direction). Based on the AR values in [3], the ratio of $\mathrm{C}_{\mathrm{H}}$ to $\mathrm{C}_{\mathrm{V}}$ in $0.13 \mu \mathrm{m}$ technology can be expressed as follows:

$$
\mathrm{AR}=3.0, \mathrm{C}_{\mathrm{H}} / \mathrm{C}_{\mathrm{V}}=3.0(\min ), 9.0(\max )
$$

As shown above, the horizontal line-to-line capacitance is three to nine times larger than the vertical line-to-line capacitance. Therefore, increasing the lateral line-to-line space is considered to be most effective.

We assume that our techniques are based on the grid-based model and the reserved layer model [16] that are generally used in automatic place and route methodology for today's large-scale SoC layouts. Therefore, to increase the lateral line-to-line space to reduce crosstalk, widening the routing grid space on the same layer seems to be the most attractive 
approach. Because the grid space is calculated according to design rules, the smallest value is generally adopted for the grid space to increase the routing resources. However, if only this approach is employed, a shortage of routing resources results in an extraordinary increase in runtime or violations remaining at the detailed route stage. Especially, if employing the highest cell utilization ratio, the situation is still worse.

The objective of this study is to address the trade-off between crosstalk noise reduction and the shortage of routing resources. We propose two novel physical design techniques, one is optimizing 3-D configuration of the routing grid, and the other is optimizing the cell utilization ratio and the routing grid space simultaneously. The details of these techniques are elaborated in the subsequent sections.

\section{A. Optimization for 3-D Configuration of the Routing Grid}

To reduce the crosstalk of a layer that has many parallel segments of wire is an effective way of reducing the overall crosstalk. For this purpose, we increase the routing grid space for the automatic place and router. This technique has two features as follows:

(I) Increasing an individual layer's routing grid space in an incremental manner.

(II) In addition to (I), choosing appropriate layers for grid space widening by considering the trade-off between crosstalk noise reduction and routing resources.

These concepts are illustrated in Fig. 1. In this four metal layer example, we can control the widening of the grid space of $\mathrm{Ma}, \mathrm{Mb}, \mathrm{Mc}$ and $\mathrm{Md}$ independently. The algorithm for this technique is described in Fig. 2.

In this flow, various types of routing grid settings that vary the choice of layers (PT_LC) and the routing grid space (PT_GS) are prepared first. After executing the route for all patterns, several analyses are performed. First we count the number of crosstalk-violated nets $(\mathrm{CN})$ for which the peak value exceeds the threshold. Secondly, the longest path delay (PD) and the runtime (RT) at the routing stage are measured. If RT exceeds the runtime threshold (TH RT), we assume that it is impossible to complete the routing. After these analyses, a cost function (COST) is introduced to find the optimum routing grid settings (OPT_LC, OPT_GS) for minimizing the crosstalk effects within a reasonable runtime. We define COST as follows.

$$
\mathrm{COST}=\mathrm{CN}^{\alpha} \mathrm{PD}^{\beta} \mathrm{RT}^{\gamma}
$$

Here, $\alpha, \beta$ and $\gamma$ are weighting factors. The correlation between crosstalk noise, runtime and the routing grid settings can be checked for various patterns of the chosen layer and routing grid space. After that, the optimum grid settings (OPT_LC, OPT_GS) for the lowest COST value are obtained.

\section{B. Co-optimization for Placement and the Routing Grid}

Another approach to obtain the routing resources is to reduce cell congestion. For this purpose, we reduce the cell utilization ratio for the automatic place and router in accordance with widening the routing grid space. This technique has two features as follows:

(I) Preparing various types of placement data that vary the cell utilization ratio. The routing resources are equal between these data, in other words, the number of $\mathrm{X}$ direction tracks and $\mathrm{Y}$ direction tracks are equal between these data.

(II) After the routing is completed, crosstalk noise and static timing analysis are performed and as a result, the optimum cell utilization ratio can be found.

These concepts are illustrated in Fig. 3, in which both reducing the cell utilization ratio and widening the routing grid space are done simultaneously to keep the routing resource equal. The algorithm for this technique is described in Fig. 4.

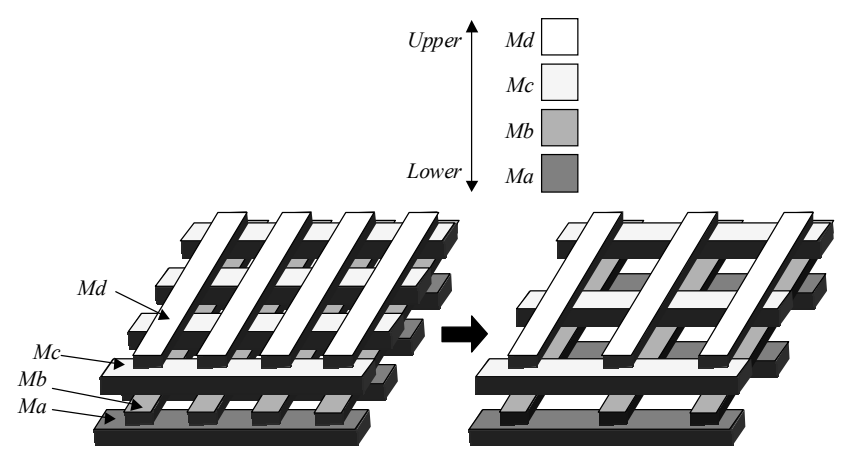

Fig. 1. Basic concept of optimization for 3-D configuration of the routing grid.

\begin{tabular}{|c|c|c|}
\hline \\
\hline \\
\hline \\
\hline & & \\
\hline & $\begin{array}{ll}\text { Inputs: } & \text { PT_LC }[i] \\
& \text {; l layer choice patterns } \\
& \text { TH_GS }[j] ; \text { grid space patterns } \\
\text { Outputs: } & \text { OPT_LC } ; \text { threshold of routing runtime } \\
\text { OPT layer choise }\end{array}$ & $\begin{array}{ll}\text { Inputs: } & \text { PT_LC }[i] \\
& \text {; layer choice patterns } \\
& \text { PT_GS }[j] ; \text {; grid space patterns } \\
& \text { TH_RT ; threshold of routing runtime }\end{array}$ \\
\hline & \multicolumn{2}{|c|}{ OPT_GS ; optimum grid space } \\
\hline \multirow[t]{4}{*}{ Variables: } & $\mathrm{CN}[\mathrm{i}, \mathrm{j}]$ & ; \#crosstalk violated net \\
\hline & $\mathrm{PD}[i, j]$ & ; longest path delay \\
\hline & $R T[i, j]$ & ; routing runtime \\
\hline & $\cos T[i, j]$ & ; cost function \\
\hline
\end{tabular}

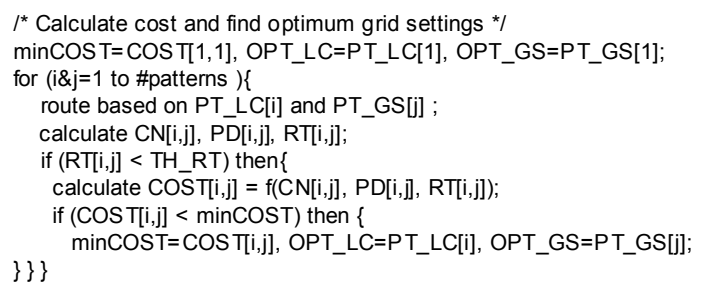

Fig. 2. Algorithm of optimization for 3-D configuration of the routing grid.

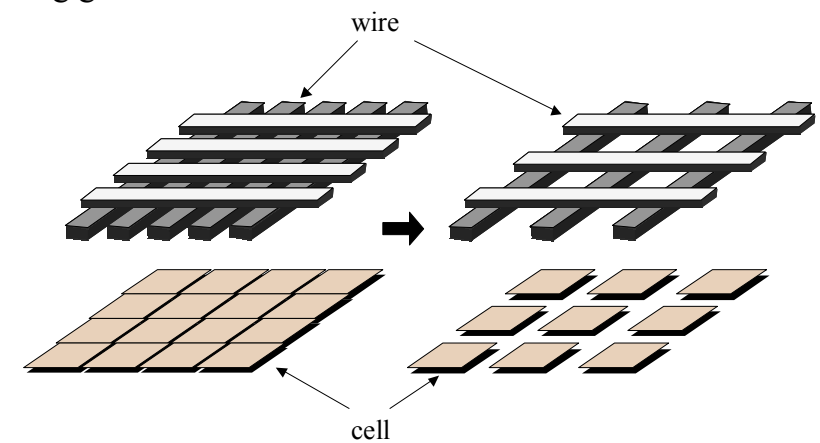

Fig. 3. Basic concept of co-optimization for placement and the routing grid. 


\section{Summary of Delay Improvement}

The maximum improvement in the delay by employing the two proposed techniques is summarized in Fig. 8. From these results, both techniques can reduce delay once the optimum cell utilization ratio and routing grid settings are obtained.

\section{Conclusions}

In this study, we demonstrated that coupling effects can be reduced by simply optimizing the parameters for automatic place and router. Experimental results showed that our two proposed techniques proved to be effective in reducing crosstalk noise within a reasonable runtime. From the experiments, the longest path delay was decreased by $15 \%$ maximum if the proposed techniques were employed. This $15 \%$ delay improvement corresponds to a half generation of process progress. Further improvement can be expected if we use both techniques simultaneously.

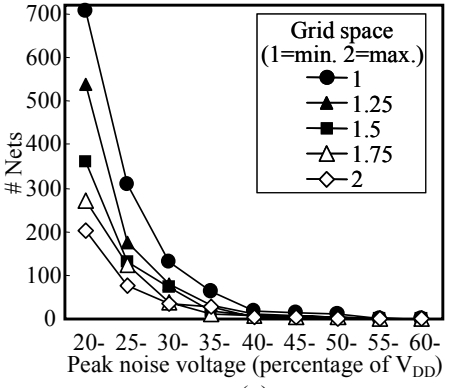

(a)

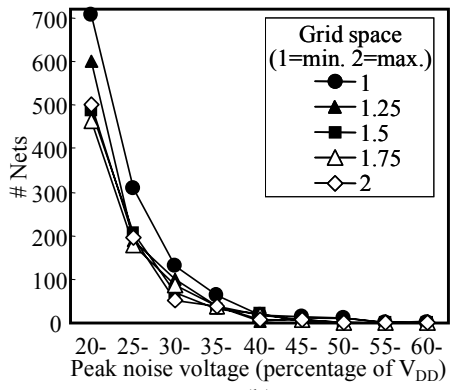

(b)
Fig. 5. Crosstalk noise vs. routing grid in (a) case1, (b) case 2.

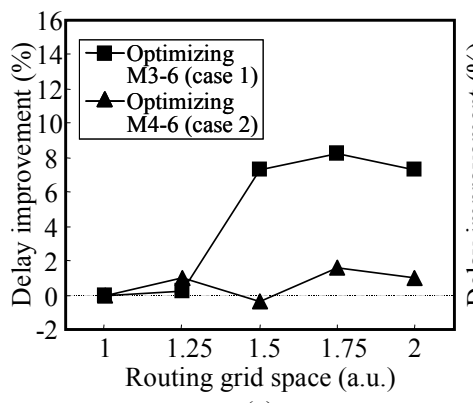

(a)

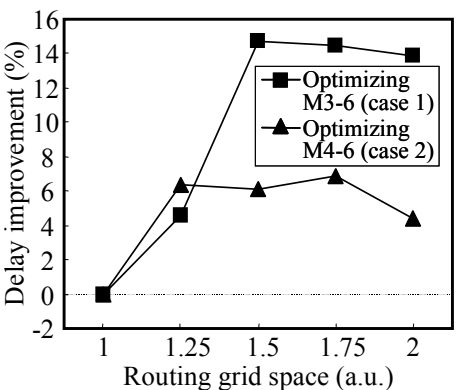

(b)
Fig. 6. Delay improvement by optimizing the 3-D configuration of routing grid, (a) Clock 1(72MHz), (b) Clock 2(114MHz).

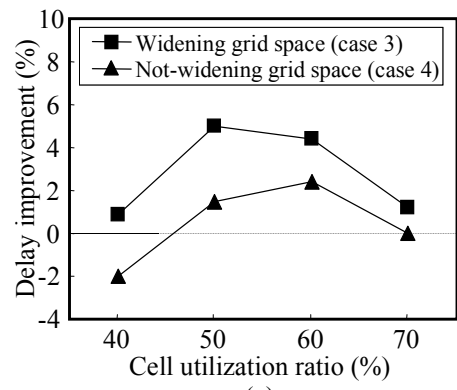

(a)

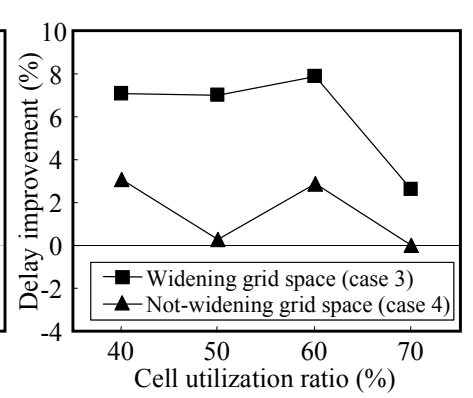

(b)
Fig. 7. Delay improvement by co-optimizing the placement and routing grid, (a) Clock 1(72MHz), (b) Clock 2(114MHz).

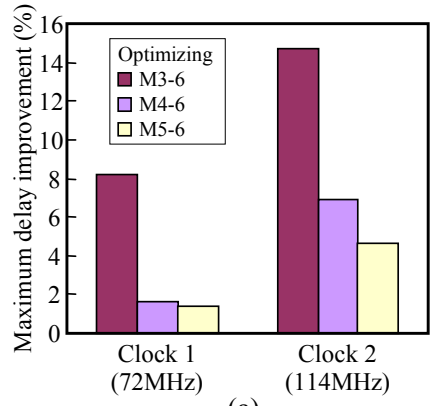

(a)

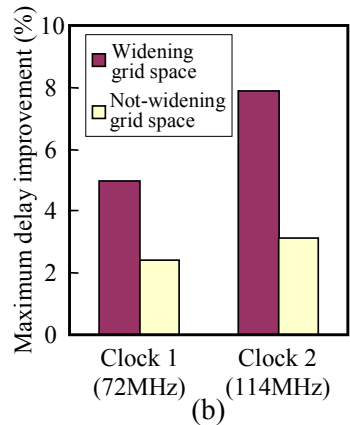

Fig. 8. Summary of Delay Improvement, (a) 3-D routing grid optimization, (b) Co-optimization.

\section{References}

[1] H. B. Bakoglu, "Circuits, Interconnections, and Packaging for VLSI," Addison-Weslay Publishing Company Inc, 1990.

[2] L. Gal, "On-Chip Cross Talk - the New Signal Integrity Challenge," Proceedings of CICC'95, pp. 251-254, 1995.

[3] J. Cong, L. He, K.-Y. Khoo, C.-K. Koh, and Pan Z., "Interconnect Design for Deep Submicron ICs," Proceedings of ICCAD'97, pp. 478-485, 1997.

[4] D. Sylvester and K. Keutzer, "Getting to the Bottom of Deep Submicron,” Proceedings of ICCAD'98, pp. 203-211, 1998.

[5] International Technology Roadmap for Semiconductors 2001 Edition, http://public.itrs.net/Files/2001ITRS/Home.htm.

[6] P. Larsson and C. Svensson, "Noise in Digital Dynamic CMOS Circuits," IEEE Journal of Solid-State Circuits, vol. 29, pp. 655-662, 1994.

[7] A. B. Kahng, S. Muddu, and D. Vidhani, "Noise and Delay Uncertainty Studies for Coupled RC Interconnects," Proceedings of IEEE ASIC/SOC conference, pp. 3-8, 1999.

[8] D. Li, A. Pua, P. Srivastava, and U. Ko, "A Repeater Optimization Methodology for Deep Sub-Micron, High-Performance Processors," Proceedings of ICCD'97, pp. 726-731, 1997.

[9] C. J. Alpert, A. Devgan, and S. T. Quay, "Buffer Insertion for Noise and Delay Optimization," Proceedings of DAC'98, pp. 362-367, 1998.

[10] A. B. Kahng, S. Muddu, E. Sarto, and R. Sharma, "Interconnect Tuning Strategies for High-Performance ICs," Proceedings of DATE'98, pp. 471-478, 1998.

[11] J. Cong, L. He, C.-K. Koh, and Z. Pan, "Global Interconnect Sizing and Spacing with Consideration of Coupling Capacitance," Proceedings of ICCAD'97, pp. 628-633, 1997.

[12] C. Nicoletta, J. Alvarez, E. Barkin, C.-C. Chao, B. R. Johnson, F. M. Lassandro, P. Patel, D. Reid, H. Sánchez, J. Siegel, M. Snyder, S. Sullivan, S. A. Taylor, and M. Vo, "A 450-MHz RISC Microprocessor with Enhanced Instruction Set and Copper Interconnect," IEEE Journal of Solid-State Circuits, pp. 1478-1491, 1999.

[13] S. P. Khatri, A. Mehrotra, R. K. Brayton, and A. Sangiovanni-Vincentelli, "A Novel VLSI Layout Fabric for Deep Sub-Micron Applications," Proceedings of DAC'99, pp. 491-496, 1999.

[14] James D. Z. Ma, and L. He, "Formulae and Applications of Interconnect Estimation Considering Shiled Insertion and Net Ordering" Proceedings of ICCAD'2001, pp. 327-332, 2001.

[15] F. Dartu and L. T. Pileggi, "Calculating Worst-Case Gate Delays Due to Dominant Capacitance Coupling," Proceedings of $D A C^{\prime}$ '97, pp. 46-51, 1997.

[16] N. Sherwani, "Algorithms for VLSI Physical Design Automation Second Edition," Kluwer Academic Publishers, 1995. 